\title{
STABLE SUBNORMS REVISITED
}

\author{
Moshe GoldBerg And W.A.J. LuxemburG
}

Let $\mathcal{A}$ be a finite-dimensional, power-associative algebra over a field $\mathbb{F}$, either $\mathbb{R}$ or $\mathbb{C}$, and let $\mathcal{S}$, a subset of $\mathcal{A}$, be closed under scalar multiplication. A real-valued function $f$ defined on $\mathcal{S}$, shall be called a subnorm if $f(a)>0$ for all $0 \neq a \in \mathcal{S}$, and $f(\alpha a)=|\alpha| f(a)$ for all $a \in \mathcal{S}$ and $\alpha \in \mathbb{F}$. If in addition, $\mathcal{S}$ is closed under raising to powers, then a subnorm $f$ shall be called stable if there exists a constant $\sigma>0$ so that

$$
f\left(a^{m}\right) \leq \sigma f(a)^{m} \quad \text { for all } a \in \mathcal{S} \text { and } m=1,2,3 \ldots
$$

The purpose of this paper is to provide an updated account of our study of stable subnorms on subsets of finite-dimensional, power-associative algebras over $\mathbb{F}$. Our goal is to review and extend several of our results in two previous papers, dealing mostly with continuous subnorms on closed sets.

\section{Introduction and review}

Let $\mathcal{A}$ be a finite-dimensional algebra over a field $\mathbb{F}$, either $\mathbb{R}$ or $\mathbb{C}$. Throughout the paper we shall assume that $\mathcal{A}$ is power-associative, that is, the subalgebra generated by any one element of $\mathcal{A}$ is associative.

Let $\mathcal{S}$ be a nonempty subset of $\mathcal{A}$, closed under scalar multiplication, namely $a \in \mathcal{S}$ and $\alpha \in \mathbb{F}$ implies $\alpha a \in \mathcal{S}$. Following [GL1], we call a function

$$
f: \mathcal{S} \rightarrow \mathbb{R}
$$

a subnorm if for all $a \in \mathcal{S}$ and $\alpha \in \mathbb{F}$,

$$
\begin{aligned}
& f(a)>0, \quad a \neq 0, \\
& f(\alpha a)=|\alpha| f(a) .
\end{aligned}
$$

If in addition, $\mathcal{S}$ is closed under raising to powers, i.e., $a \in \mathcal{S}$ implies $a^{m} \in \mathcal{S}, m=1,2,3, \ldots$, then a subnorm $f$ on $\mathcal{S}$ shall be called a submodulus if

$$
f\left(a^{m}\right)=f(a)^{m} \quad \text { for all } \quad a \in \mathcal{S} \quad \text { and } \quad m=1,2,3, \ldots .
$$

We recall that if $\mathcal{S}$ is closed under scalar multiplication and under addition, then a real-valued function $N$ on $\mathcal{S}$ is a norm if for all $a, b \in \mathcal{S}$ and 
$\alpha \in \mathbb{F}$,

$$
\begin{aligned}
& N(a)>0, \quad a \neq 0, \\
& N(\alpha a)=|\alpha| N(a), \\
& N(a+b) \leq N(a)+N(b)
\end{aligned}
$$

hence, a norm is a subadditive, continuous subnorm on $\mathcal{S}$.

Surely, a subnorm is said to be continuous if it is continuous with respect to the (unique) finite-dimensional norm-topology on $\mathcal{A}$.

Let a subset $\mathcal{S}$ of $\mathcal{A}$ be closed under scalar multiplication and under raising to powers. Following familiar terminology for norms, we say that a subnorm $f$ on $\mathcal{S}$ is stable if there exists a positive constant $\sigma$ such that

$$
f\left(a^{m}\right) \leq \sigma f(a)^{m} \quad \text { for all } \quad a \in \mathcal{S} \text { and } m=1,2,3, \ldots
$$

The purpose of this paper is to review and extend some of our results in [GL1] and [GGL], dealing mostly with continuous subnorms on closed subsets of finite-dimensional, power-associative algebras.

We begin by quoting:

Theorem 1.1 ([GL1, Theorem 1.1(a)]; [GGL, Theorem 1]). Let $\mathcal{S}, a$ closed subset of a finite-dimensional, power-associative algebra $\mathcal{A}$ over $\mathbb{F}$, be closed under scalar multiplication and under raising to powers. Let $f$ be a continuous subnorm on $\mathcal{S}$, and let $g$ be a continuous submodulus on $\mathcal{S}$. Then $f$ is stable if and only if $f \geq g$ on $\mathcal{S}$.

An elementary consequence of this theorem is:

Corollary 1.1 ([GL1, Corollary 1.1]; [GGL, Corollary 1]). If $\mathcal{A}, \mathcal{S}$, and $g$ are as in Theorem 1.1, then $g$ is the only continuous submodulus on $\mathcal{S}$.

Proof. Let $g^{\prime}$ be another submodulus on $\mathcal{S}$. Since $g$ and $g^{\prime}$ are stable subnorms, Theorem 1.1 implies $g \geq g^{\prime}$ and $g^{\prime} \geq g$, and the proof is complete.

In the absence of a submodulus, Theorem 1.1 tells us nothing. Indeed, as indicated by the following simple result, not every set has a submodulus:

Proposition 1.1 ([GGL, Proposition 3]). If $\mathcal{S}$, a subset of a power-associative algebra $\mathcal{A}$ over $\mathbb{F}$, contains nonzero nilpotent elements, then $\mathcal{S}$ has no submodulus.

Theorem 1.1 and Corollary 1.1 were illustrated both in [GL1] and [GGL] by viewing the complex numbers

$$
\mathbb{C} \equiv\{z=\alpha+i \beta: \quad \alpha, \beta \in \mathbb{R}\}
$$

as a 2-dimensional algebra over the reals, and noting that

$$
|z| \equiv \sqrt{\alpha^{2}+\beta^{2}}
$$


is a continuous submodulus. Thus, by Corollary 1.1, the absolute-value function in (1.1) is the only continuous submodulus on $\mathbb{C}$; and by Theorem 1.1, a continuous subnorm $f$ on $\mathbb{C}$ is stable if and only if $f(z) \geq|z|$ for all $z \in \mathbb{C}$. For instance, observing that for each fixed $p, 0<p \leq \infty$,

$$
|z|_{p} \equiv\left(|\alpha|^{p}+|\beta|^{p}\right)^{1 / p}, \quad z=\alpha+i \beta \in \mathbb{C},
$$

is a continuous subnorm on $\mathbb{C}$ (a norm if and only if $1 \leq p \leq \infty$ ), we find that $|\cdot|_{p}$ is stable on $\mathbb{C}$ if and only if $0<p \leq 2$.

Similar examples in $[\mathbf{G L 1}]$ and $[\mathbf{G G L}]$ concerned the non-commutative, 4-dimensional, associative algebra of the quaternions,

$$
\mathbb{H} \equiv\{q=\alpha+i \beta+j \gamma+k \delta: \quad \alpha, \beta, \gamma, \delta \in \mathbb{R}\}, \quad i^{2}=j^{2}=k^{2}=i j k=-1,
$$

with its continuous submodulus

$$
|q| \equiv \sqrt{\alpha^{2}+\beta^{2}+\gamma^{2}+\delta^{2}}
$$

and the 8-dimensional, power-associative algebra of the octonions (often called the Cayley numbers or the octaves),

$$
\mathbb{O} \equiv\left\{c=\gamma_{1}+e_{2} \gamma_{2}+\cdots+e_{8} \gamma_{8}: \quad \gamma_{i} \in \mathbb{R}\right\}
$$

with its intricate multiplication rule (e.g., $[\mathbf{B}]$ ) and its continuous submodulus

$$
|c| \equiv \sqrt{\gamma_{1}^{2}+\cdots+\gamma_{8}^{2}} .
$$

With Theorem 1.1 in mind, we remark that if our set is closed and consists only of nilpotents, then the question of stability becomes a triviality:

Proposition 1.2 ([GGL, Proposition 4]). Let $\mathcal{S}$ be a closed set of nilpotent elements in a finite-dimensional, power-associative algebra, closed under scalar multiplication and under raising to powers. Then any continuous subnorm on $\mathcal{S}$ is stable.

Another simple observation is given by the following assertion which may prove useful in investigating stability, even when a submodulus does not exist:

Proposition 1.3 ([GGL, Proposition 5]). Let $\mathcal{S}$, a closed subset of a finitedimensional, power-associative algebra $\mathcal{A}$ over $\mathbb{F}$, be closed under scalar multiplication and under raising to powers. Let $f$ and $g$ be continuous subnorms on $\mathcal{S}$ such that $f$ is stable and $f \leq g$. Then $g$ is stable on $\mathcal{S}$.

If $\mathcal{S}$, a subset of $\mathbb{F}^{n \times n}$, the algebra of $n \times n$ matrices over $\mathbb{F}$, is void of nonzero nilpotent matrices, then obviously, the spectral radius,

$$
\rho(A) \equiv \max \{|\lambda|: \quad \lambda \text { eigenvalue of } A\}, \quad A \in \mathcal{S},
$$

is a submodulus on $\mathcal{S}$. This fact, combined with Proposition 1.1 and Corollary 1.1, immediately produce: 
Theorem 1.2 ([GL1, Theorem 1.2]; [GGL, Proposition 6]). Let $\mathcal{S}$, a subset of $\mathbb{F}^{n \times n}$, be closed under scalar multiplication and under raising to powers. Then:

(a) $\mathcal{S}$ has a submodulus if and only if $\mathcal{S}$ has no nonzero nilpotent matrices.

(b) If $\mathcal{S}$ has no nonzero nilpotents, then $\rho$, the spectral radius, is a submodulus on $\mathcal{S}$.

(c) If $\mathcal{S}$ is closed and has no nonzero nilpotents, then $\rho$ is the only continuous submodulus on $\mathcal{S}$.

We note that if $\mathcal{S}$ in Theorem 1.2 is not closed, then $\mathcal{S}$ may have infinitely many continuous submoduli. For example, let $\mathcal{S}$ be the class of all $n \times n$ invertible matrices over $\mathbb{F}$. Evidently, this open set is closed under scalar multiplication and under raising to powers, and is void of nonzero nilpotents. Put

$$
\tau(A) \equiv \min \{|\lambda|: \quad \lambda \text { eigenvalue of } A\}, \quad A \in \mathcal{S},
$$

and observe that $\tau$ is a positive continuous function on $\mathcal{S}$, satisfying for all $\alpha \in \mathbb{F}, A \in \mathcal{S}$, and positive integers $m$,

$$
\begin{aligned}
\tau(\alpha A) & =|\alpha| \tau(A), \\
\tau\left(A^{m}\right) & =\tau(A)^{m} .
\end{aligned}
$$

Hence,

$$
g_{\kappa}(A) \equiv \rho(A)^{\kappa+1} \tau(A)^{-\kappa}
$$

is a continuous submodulus on $\mathcal{S}$ for every real constant $\kappa$.

If $\mathcal{S}$ in Theorem 1.2 is not closed, we also observe that $\mathcal{S}$ may have infinitely many discontinuous submoduli. An example supporting this statement was given in [GGL] with $\mathcal{S}$ the class of all $n \times n$ normal matrices over $\mathbb{F}$. Since this closed set is closed under scalar multiplication and under raising to powers, and since it is void of nonzero nilpotents, Theorem 1.2(c) tells us that the only continuous submodulus on $\mathcal{S}$ is the spectral radius. It is not hard to see, however, that

$$
h_{\kappa}(A) \equiv \begin{cases}\rho(A)^{\kappa+1} \tau(A)^{-\kappa}, & \tau(A) \neq 0, \\ \rho(A), & \tau(A)=0,\end{cases}
$$

is a submodulus on $\mathcal{S}$ for every real constant $\kappa$. As expected, if $\kappa \neq 0$, then $h_{\kappa}$ is discontinuous, since by appealing to the normal matrix

$$
A_{\varepsilon}=\operatorname{diag}(1, \ldots, 1, \varepsilon), \quad \varepsilon>0,
$$

we get

$$
\lim _{\varepsilon \rightarrow 0} h_{\kappa}\left(A_{\varepsilon}\right)= \begin{cases}\infty, & \kappa>0, \\ 0, & \kappa<0,\end{cases}
$$

whereas $h_{\kappa}\left(A_{0}\right)=1$. Other examples of a similar nature can be found in [GL2]. 
Dealing with matrices, we follow standard nomenclature and call a subnorm $f$ on a subset $\mathcal{S}$ of $\mathbb{F}^{n \times n}$ spectrally dominant if $f$ majorizes the spectral radius, i.e.,

$$
f(A) \geq \rho(A) \text { for all } A \in \mathcal{S} .
$$

Hence, Theorems 1.1 and 1.2(c) yield:

Theorem 1.3 ([GL1, Theorem 1.3(a)]; [GGL, Corollary 4]). Let $\mathcal{S}, a$ closed subset of $\mathbb{F}^{n \times n}$, be closed under scalar multiplication and under raising to powers. If $\mathcal{S}$ contains no nonzero nilpotent matrices and $f$ is a continuous subnorm on $\mathcal{S}$, then $f$ is stable if and only if it is spectrally dominant on $\mathcal{S}$.

It is not hard to see that half of Theorem 1.3 can be obtained without assuming that $\mathcal{S}$ contains no nonzero nilpotents:

Proposition 1.4 ([GL2, Theorem 1.5(b)]; [GGL, Proposition 7]). Let $\mathcal{S}$, a closed subset of $\mathbb{F}^{n \times n}$, be closed under scalar multiplication and under raising to powers, and let $f$ be a continuous, stable subnorm on $\mathcal{S}$. Then $f$ is spectrally dominant on $\mathcal{S}$.

As for the other half of Theorem 1.3, we claim that the assumption that $\mathcal{S}$ contains no nonzero nilpotents cannot be dropped. We shall prove this assertion (compare [GGL]) by exhibiting a subalgebra $\mathcal{A}$ of $\mathbb{F}^{n \times n}$ that contains nonzero nilpotents as well as elements which are not nilpotent, and a norm that is spectrally dominant but not stable on $\mathcal{A}$.

Indeed, let $I$ be the $n \times n$ identity matrix, and let $\mathcal{U}_{n}$ denote the class of strictly upper triangular matrices in $\mathbb{F}^{n \times n}$. Then

$$
\mathcal{A} \equiv\left\{\alpha I+B: \quad \alpha \in \mathbb{F}, \quad B \in \mathcal{U}_{n}\right\}
$$

is a subalgebra of $\mathbb{F}^{n \times n}$ of the desired type. Let $N$ be a norm on $\mathcal{U}_{n}$ and define

$$
N^{\prime}(A) \equiv \max \{|\alpha|, N(B)\}, \quad A=\alpha I+B \in \mathcal{A}, \quad B \in \mathcal{U}_{n} .
$$

Evidently, $N^{\prime}$ is a spectrally dominant norm on $\mathcal{A}$, so it remains to show that $N^{\prime}$ is unstable. Consider

$$
A_{\kappa}=I+J_{\kappa} \in \mathcal{A}
$$

where $J_{\kappa}$ is the $n \times n$ matrix whose upper-right entry is a constant $\kappa$ and all other entries vanish. Fix $\kappa$ so that $N\left(J_{\kappa}\right)=1$; hence $N^{\prime}\left(A_{\kappa}\right)=1$. On the other hand, since $J_{\kappa}^{m}=0$ for all $m \geq 2$, we have

$$
A_{\kappa}^{m}=\left(I+J_{\kappa}\right)^{m}=I+m J_{\kappa}, \quad m=1,2,3, \ldots .
$$

So, as

$$
N\left(m J_{\kappa}\right)=m N\left(J_{\kappa}\right)=m \underset{m \rightarrow \infty}{\longrightarrow} \infty
$$

we obtain

$$
N^{\prime}\left(A_{\kappa}^{m}\right)=N^{\prime}\left(I+m J_{\kappa}\right)=\max \left\{1, N\left(m J_{\kappa}\right)\right\} \underset{m \rightarrow \infty}{\longrightarrow} \infty
$$


and our assertion holds.

\section{Existence and uniqueness of submoduli}

Theorem 1.2 provides a rather complete account regarding the existence and uniqueness of submoduli on sets of matrices. As we shall see, this theorem can be adapted almost verbatim to describe the situation for arbitrary finitedimensional, associative algebras over $\mathbb{F}$.

Verily, if $\mathcal{A}$ is a finite-dimensional, associative algebra over $\mathbb{F}$, it is well known that every element $a \in \mathcal{A}$ possesses a unique minimal polynomial, i.e., a monic $p_{a}$ of positive degree, with coefficients in $\mathbb{F}$, satisfying $p_{a}(a)=0$ and dividing every other polynomial that annihilates $a$. Hence, for each $a \in \mathcal{A}$, we may define

$$
\rho^{\prime}(a) \equiv \max \left\{|\lambda|: \quad \lambda \text { root of } p_{a}\right\} .
$$

Since $\mathcal{A}$ is associative, it is algebraically isomorphic to a matrix algebra over $\mathbb{F}$, where the operations (scalar multiplication, addition and multiplication) and the topology are preserved. Let $\varphi$ be such an isomorphism and denote the corresponding matrix algebra by $\mathcal{A}_{\varphi}$, so that

$$
\varphi: \mathcal{A} \rightarrow \mathcal{A}_{\varphi} ; \quad \varphi(a) \equiv A_{a} \in \mathcal{A}_{\varphi}, \quad a \in \mathcal{A} .
$$

It follows that if $a$ is an element in $\mathcal{A}$ with minimal polynomial $p_{a}$, then $p_{a}$ is the minimal polynomial of the corresponding matrix $A_{a}=\varphi(a)$. So, $\rho^{\prime}(a)$ and the spectral radius of $A_{a}$ coincide, i.e.,

$$
\rho^{\prime}(a)=\rho(\varphi(a))=\rho\left(A_{a}\right) .
$$

Using the definition of $\rho^{\prime}$ in (2.1), and the common properties of the isomorphism $\varphi$ and the spectral radius on $\mathcal{A}_{\varphi}$, we readily find that for every $a \in \mathcal{A}$ and $\alpha \in \mathbb{F}$,

$$
\begin{aligned}
& \rho^{\prime}(a) \geq 0, \\
& \rho^{\prime}(\alpha a)=|\alpha| \rho^{\prime}(a), \\
& \rho^{\prime}\left(a^{m}\right)=\rho^{\prime}(a)^{m}, \quad m=1,2,3, \ldots .
\end{aligned}
$$

Further, we obtain that $\rho^{\prime}$ is a continuous function on $\mathcal{A}$ that vanishes only on nilpotent elements.

Assisted by this argument and by Corollary 1.1, we are now ready to post the following generalization of Theorem 1.2:

Theorem 2.1 (compare [GGL, Theorem 2]). Let $\mathcal{S}$, a subset of a finitedimensional, associative algebra $\mathcal{A}$ over $\mathbb{F}$, be closed under scalar multiplication and under raising to powers. Then:

(a) $\mathcal{S}$ has a submodulus if and only if $\mathcal{S}$ has no nonzero nilpotent elements.

(b) If $\mathcal{S}$ has no nonzero nilpotents then the continuous function $\rho^{\prime}$ defined in (2.1), is a submodulus on $\mathcal{S}$. 
(c) If $\mathcal{S}$ is closed and has no nonzero nilpotents, then $\rho^{\prime}$ is the only continuous submodulus on $\mathcal{S}$.

Falling back on our last argument, we comment that if $f$ is a subnorm (even a submodulus or a norm) on the subset $\mathcal{S}$ described in Theorem 2.1, and if $\varphi$ is the isomorphism in (2.2), then $f_{\varphi}$, defined by

$$
f_{\varphi}\left(A_{a}\right) \equiv f(a), \quad a \in \mathcal{S},
$$

is a subnorm (submodulus, norm) on the set $\mathcal{S}_{\varphi}$, the image of $\mathcal{S}$ under $\varphi$, and vice versa. Moreover, it is not hard to see that $f$ will be continuous or stable on $\mathcal{S}$ if and only if $f_{\varphi}$ will be so on $\mathcal{S}_{\varphi}$.

Hence, we can register the following analogue of Proposition 1.4:

Proposition 2.1. Let $\mathcal{S}$, a closed subset of a finite-dimensional, associative algebra $\mathcal{A}$ over $\mathbb{F}$, be closed under scalar multiplication and under raising to powers, and let $f$ be a continuous, stable subnorm on $\mathcal{S}$. Then $f$ majorizes $\rho^{\prime}$ on $\mathcal{S}$.

To illustrate Theorem 2.1, let us revisit the algebra of complex numbers

$$
\mathbb{C} \equiv\{z=\alpha+i \beta: \quad \alpha, \beta \in \mathbb{R}\}
$$

and note that the minimal polynomial of $z \in \mathbb{C}$ (over the reals) is

$$
p_{z}(\lambda)=\lambda^{2}-2 \alpha \lambda+\alpha^{2}+\beta^{2}, \quad z=\alpha+i \beta .
$$

Since the roots of $p_{z}$ are $z$ and $\bar{z}$, it follows that

$$
\rho^{\prime}(z)=|z| \text {. }
$$

Furthermore, as $\mathbb{C}$ contains no nonzero nilpotents, Theorem 2.1(c) implies the previously mentioned fact (see (1.1)) that $\rho^{\prime}$ in (2.5) is the only continuous submodulus on $\mathbb{C}$.

As was shown in [GL1] and [GGL], (2.5) can be also obtained by considering the familiar isomorphism

$$
z \rightarrow A_{z} \equiv\left(\begin{array}{cc}
\alpha & \beta \\
-\beta & \alpha
\end{array}\right), \quad z=\alpha+i \beta \in \mathbb{C},
$$

that maps $\mathbb{C}$ onto the 2-dimensional subalgebra of $\mathbb{R}^{2 \times 2}$,

$$
\mathcal{A}_{2}(\mathbb{R}) \equiv\left\{\left(\begin{array}{cc}
\alpha & \beta \\
-\beta & \alpha
\end{array}\right): \quad \alpha, \beta \in \mathbb{R}\right\} .
$$

Referring to (2.3), we obtain

$$
\rho^{\prime}(z)=\rho\left(\begin{array}{cc}
\alpha & \beta \\
-\beta & \alpha
\end{array}\right)=\sqrt{\alpha^{2}+\beta^{2}}=|z|, \quad z=\alpha+i \beta \in \mathbb{C},
$$

and (2.5) follows.

When $\mathcal{A}$ is merely power-associative, the question of existence and especially uniqueness of a submodulus is more demanding, and it seems that only parts (a) and (b) of Theorem 2.1 can be salvaged. 
We begin our examination of this case by observing that power-associativity is enough to force the existence of a minimal polynomial for every element of $\mathcal{A}$; hence the definition of $\rho^{\prime}$ in (2.1) remains valid. Now let $a \in \mathcal{A}$ be an arbitrary element, and let $\mathcal{A}_{a}$ denote the subalgebra of $\mathcal{A}$ generated by $a$. Since $\mathcal{A}$ is power-associative, $\mathcal{A}_{a}$ is associative; so by the argument preceding Theorem 2.1, we find that for every $a \in \mathcal{A}$ and $\alpha \in \mathbb{F}, \rho^{\prime}$ satisfies the relations in (2.4), and $\rho^{\prime}(a)=0$ if and only if $a$ is nilpotent. Therefore, in analogy with the first two parts of Theorem 2.1, we may record:

Theorem 2.2. Let $\mathcal{S}$, a subset of a finite-dimensional, power-associative algebra $\mathcal{A}$ over $\mathbb{F}$, be closed under scalar multiplication and under raising to powers. Then:

(a) $\mathcal{S}$ has a submodulus if and only if $\mathcal{S}$ has no nonzero nilpotent elements.

(b) If $\mathcal{S}$ has no nonzero nilpotents then $\rho^{\prime}$ defined in (2.1), is a submodulus on $\mathcal{S}$.

As it stands, we are unable to determine whether, in the power-associative case, $\rho^{\prime}$ is always continuous on $\mathcal{A}$. This drawback can be mended if one is willing to assume more than just power-associativity; for example, that $\mathcal{A}$ is an alternative algebra, i.e., that the subalgebra generated by any two elements in $\mathcal{A}$ is associative.

Assuming that $\mathcal{A}$ is alternative, consider the regular left representation of $\mathcal{A}$, where with each element $a \in \mathcal{A}$ we associate the mapping $T_{a}$ defined by

$$
T_{a} x=a x \text { for all } x \in \mathcal{A} .
$$

Since $T_{a}$ is a linear transformation on a finite-dimensional vector space, its spectral radius,

$$
\rho\left(T_{a}\right) \equiv \max \left\{|\lambda|: \quad \lambda \text { eigenvalue of } T_{a}\right\},
$$

is well defined. Set

$$
\rho^{\prime \prime}(a) \equiv \rho\left(T_{a}\right), \quad a \in \mathcal{A} .
$$

Then surely, for every $a \in \mathcal{A}$ and $\alpha \in \mathbb{F}, \rho^{\prime \prime}$ satisfies

$$
\begin{aligned}
& \rho^{\prime \prime}(a) \geq 0, \\
& \rho^{\prime \prime}(\alpha a)=|\alpha| \rho^{\prime \prime}(a) .
\end{aligned}
$$

Moreover, $\rho^{\prime \prime}$ is continuous on $\mathcal{A}$ since the matrix coefficients of $T_{a}$ relative to any basis of $\mathcal{A}$ are continuous.

Now, let $a$ and $x$ be any two elements in $\mathcal{A}$. Since $\mathcal{A}$ is alternative, the subalgebra of $\mathcal{A}$ generated by $a$ and $x$ is associative. Hence, by the definition of $T_{a}$ in (2.6), for every positive integer $m$, we obtain

$$
T_{a^{m}}=T_{a}^{m} .
$$


Consequently, we get

$$
\rho^{\prime \prime}\left(a^{m}\right)=\rho^{\prime \prime}(a)^{m}, \quad m=1,2,3, \ldots ;
$$

and further, we maintain that an element $a \in \mathcal{A}$ is nilpotent if and only if $\rho^{\prime \prime}(a)=0$. Indeed, if $a$ is nilpotent, then $a^{k}=0$ for some positive integer $k$. Thus $T_{a^{k}}=0$; so by (2.8),

$$
\rho\left(T_{a}\right)^{k}=\rho\left(T_{a}^{k}\right)=\rho\left(T_{a^{k}}\right)=0,
$$

whence $\rho^{\prime \prime}(a)=\rho\left(T_{a}\right)=0$.

Conversely, if $\rho^{\prime \prime}(a)=0$, then $\rho\left(T_{a}\right)=0$; so $T_{a}$ is nilpotent, that is, $T_{a}^{k}=0$ for some $k$. By (2.8) therefore, $T_{a^{k}}=0$, or in other words, $a^{k} x=0$ for all $x \in \mathcal{A}$. Hence, $a^{k+1}=0$, and our claim follows.

With this discussion, and by Corollary 1.1, we have thus established:

Theorem 2.3. Let $\mathcal{S}$, a subset of a finite-dimensional, alternative algebra $\mathcal{A}$ over $\mathbb{F}$, be closed under scalar multiplication and under raising to powers. Then:

(a) $\mathcal{S}$ has a submodulus if and only if $\mathcal{S}$ has no nonzero nilpotent elements.

(b) If $\mathcal{S}$ has no nonzero nilpotents then the continuous function $\rho^{\prime \prime}$ defined in (2.7), is a submodulus on $\mathcal{S}$.

(c) If $\mathcal{S}$ is closed and has no nonzero nilpotents, then $\rho^{\prime \prime}$ is the only continuous submodulus on $\mathcal{S}$.

We recall (e.g., [B, Theorem 2]) that the octonions constitute an alternative algebra. However, knowing already that the only continuous submodulus on $\mathbb{O}$ is the absolute value function in (1.2), all we can learn from Theorem 2.3 about the octonions is that for each $c \in \mathbb{O}$, the spectral radius of the left regular representation $T_{c}$, is $|c|$.

Note that if $\mathcal{A}$ is associative, then $\rho^{\prime}$ in (2.1) and $\rho^{\prime \prime}$ in (2.7) coincide. This is so since in the associative case, the roots of $p_{a}$, the minimal polynomial of an element $a \in \mathcal{A}$, are those of the characteristic polynomial of the left representation $T_{a}$ in (2.6).

\section{Stable norms on $\mathbb{R}^{n \times n}$}

Motivated by Theorem 1.3, we tried, without a shred of success, to characterize the class of all matrix algebras over $\mathbb{F}$ that contain nonzero nilpotents, with the property that a norm on such an algebra will be stable if and only if it is spectrally dominant.

The renowned Friedland-Zenger theorem tells us that $\mathbb{C}^{n \times n}$, the algebra of $n \times n$ complex matrices, belongs to this class, namely:

Theorem 3.1 ([FZ, Theorem 1]). A norm on $\mathbb{C}^{n \times n}$ is stable if and only if it is spectrally dominant. 
In [GGL] we conjectured that $\mathbb{R}^{n \times n}$ also belongs to this class. Unable to prove this conjecture, we settled for a simpler result about absolute norms. Recalling that a norm $N$ on $\mathbb{F}^{n \times n}$ is absolute if

$$
N(A)=N\left(A^{+}\right), \quad A \in \mathbb{F}^{n \times n},
$$

where $A^{+}$is the matrix obtained by taking the absolute values of the entries of $A$, we proved:

Theorem 3.2 ([GGL, Theorem 5]). An absolute norm on $\mathbb{R}^{n \times n}$ is stable if and only if it is spectrally dominant.

Theorem 3.2 can be easily strengthened by recalling (e.g., [G2]) that a norm $N$ on $\mathbb{F}^{n \times n}$ is quasimonotone if it is monotone on the cone of matrices with positive entries; i.e.,

$$
0 \leq A \leq B \Rightarrow f(A) \leq f(B), \quad A, B \in \mathbb{F}^{n \times n},
$$

where the inequalities $0 \leq A \leq B$ are construed entrywise.

With this definition we are led to:

Theorem 3.3. A quasimonotone norm on $\mathbb{R}^{n \times n}$ is stable if and only if it is spectrally dominant.

Proof. The proof is almost identical to that of Theorem 5 in [GGL]. If $N$ is a stable norm on $\mathbb{R}^{n \times n}$, then by Proposition $1.4, N$ is spectrally dominant.

Conversely, let $N$ be a quasimonotone, spectrally dominant norm on $\mathbb{R}^{n \times n}$. Extend $N$ to $\mathbb{C}^{n \times n}$ by putting

$$
N^{\prime}(A) \equiv N\left(A^{+}\right), \quad A \in \mathbb{C}^{n \times n} .
$$

As $N$ is quasimonotone, it follows that $N^{\prime}$ is a norm on $\mathbb{C}^{n \times n}$ since

$$
\begin{aligned}
& N^{\prime}(A)>0, \quad A \neq 0, \\
& N^{\prime}(\alpha A)=|\alpha| N^{\prime}(A), \quad \alpha \in \mathbb{C}, \quad A \in \mathbb{C}^{n \times n},
\end{aligned}
$$

and for all $A, B \in \mathbb{C}^{n \times n}$,

$$
\begin{aligned}
N^{\prime}(A+B) & =N\left((A+B)^{+}\right) \\
& \leq N\left(A^{+}+B^{+}\right) \\
& \leq N\left(A^{+}\right)+N\left(B^{+}\right) \\
& =N^{\prime}(A)+N^{\prime}(B) .
\end{aligned}
$$

Now, an old result of Frobenius (e.g., [W], [HJ, Theorem 8.1.18] and [G1, Example 1]) maintains that $\rho$, the spectral radius, is semimonotone on $\mathbb{C}^{n \times n}$; that is, if $B$ and $C$ are $n \times n$ matrices with $B \geq C^{+}$, then

$$
\rho(B) \geq \rho(C) \text {. }
$$

It follows that

$$
N^{\prime}(A)=N\left(A^{+}\right) \geq \rho\left(A^{+}\right) \geq \rho(A), \quad A \in \mathbb{C}^{n \times n},
$$


hence $N^{\prime}$ is spectrally dominant on $\mathbb{C}^{n \times n}$. By the Friedland-Zenger theorem, therefore, $N^{\prime}$ is stable on $\mathbb{C}^{n \times n}$; hence $N$, the restriction of $N^{\prime}$ to the real $n \times n$ matrices, is stable on $\mathbb{R}^{n \times n}$.

With Theorem 3.3 at hand, recall that a norm $N$ on $\mathbb{F}^{n \times n}$ is monotone if

$$
A^{+} \leq B^{+} \Rightarrow N(A) \leq N(B), \quad A, B \in \mathbb{F}^{n \times n} ;
$$

so monotonicity implies quasimonotonicity. Further, a well-known result ([Z, Theorem 108.1], [HJ, Theorem 5.5.10]; compare [BSW]) states that a norm $N$ on $\mathbb{F}^{n \times n}$ is monotone if and only if it is absolute. Thus, Theorem 3.3 is indeed an extension of Theorem 3.2. This is a nontrivial extension, as some of the best known norms on $\mathbb{F}^{n \times n}$ are quasimonotone but not monotone ([G1] and $[\mathbf{G 2}])$; e.g., the numerical radius

$$
r(A) \equiv \max \left\{\left|x^{*} A x\right|: \quad x \in \mathbb{C}^{n}, x^{*} x=1\right\}, \quad A \in \mathbb{F}^{n \times n},
$$

and the $\ell_{p}$ operator norms for $1<p<\infty$,

$$
\|A\|_{p} \equiv \max \left\{|A x|_{p}: \quad x \in \mathbb{C}^{n},|x|_{p}=1\right\}, \quad A \in \mathbb{F}^{n \times n},
$$

where

$$
|x|_{p} \equiv\left(\sum_{j=1}^{n}\left|\xi_{j}\right|^{p}\right)^{1 / p}, \quad x=\left(\xi_{j}\right), \quad(n \geq 2) .
$$

While Theorem 3.3 excludes norms that are not qusimonotone, we remark that there is a whole universe of such norms, some of which are easy to come by. In fact, in order to display a non-quasimonotone norm on $\mathbb{F}^{n \times n}$, it suffices to construct such a norm on $\mathbb{F}^{n}$. For let $|\cdot|$ be a non-quasimonotone norm on $\mathbb{F}^{n}$, so we can find vectors $x_{0}, y_{0} \in \mathbb{F}^{n}$ with $0 \leq x_{0} \leq y_{0}$ and $\left|x_{0}\right|>\left|y_{0}\right|$. For $A \in \mathbb{F}^{n \times n}$, write $A=\left(a_{1}, \ldots, a_{n}\right)$ where $a_{1}, \ldots, a_{n}$ are the columns of $A$, and set

$$
\|A\| \equiv \max _{1 \leq j \leq n}\left|a_{j}\right|
$$

Then $\|\cdot\|$ is obviously a norm on $\mathbb{F}^{n \times n}$. Moreover, putting

$$
A_{0}=\left(x_{0}, \ldots, x_{0}\right), \quad B_{0}=\left(y_{0}, \ldots, y_{0}\right),
$$

we get $0 \leq A_{0} \leq B_{0}$ and $\left\|A_{0}\right\|>\left\|B_{0}\right\|$; hence $\|\cdot\|$ is not quasimonotone.

To illustrate the construction above (compare [G2]) consider the matrix

$$
H=\left(\begin{array}{cc}
2 & -1 \\
-1 & 1
\end{array}\right)
$$

Since $H$ is positive definite, it follows that

$$
(x, y)_{H} \equiv y^{T} H x, \quad x, y \in \mathbb{R}^{2},
$$

( $T$ denoting the transpose) is an inner product on $\mathbb{R}^{2}$; thus

$$
|x|_{H} \equiv(x, x)_{H}^{1 / 2}=\left(2 \xi_{1}^{2}+\xi_{2}^{2}-2 \xi_{1} \xi_{2}\right)^{1 / 2}, \quad x=\left(\xi_{1}, \xi_{2}\right)^{T},
$$


is a norm on the same space. Further, the column vectors $x_{0}=(1,0)^{T}, y_{0}=$ $(1,1)^{T}$, satisfy $0 \leq x_{0} \leq y_{0},\left|x_{0}\right|_{H}>\left|y_{0}\right|_{H} ;$ so $|\cdot|_{H}$ is not quasimonotone on $\mathbb{R}^{2}$, and the corresponding matrix norm,

$$
\|A\|_{H} \equiv \max \left\{\left|a_{1}\right|_{H},\left|a_{2}\right|_{H}\right\}, \quad A=\left(a_{1}, a_{2}\right),
$$

is not quasimonotone on $\mathbb{R}^{2 \times 2}$.

Appealing to the $2 \times 2$ matrix $E$ all whose entries are 1 , we see that the norm in (3.1) is not spectrally dominant. Not surprisingly, $\|\cdot\|_{H}$ is not stable either, since

$$
\|E\|_{H}=1, \quad\left\|E^{m}\right\|_{H}=2^{m-1}\|E\|_{H}=2^{m-1} \underset{m \rightarrow \infty}{\longrightarrow} \infty .
$$

In concluding this paper, we point out that the Friedland-Zenger theorem cannot be extended to proper subnorms, i.e., to subnorms which are not norms. Indeed, let $f$ be a subnorm (even a norm) on $\mathbb{C}^{n \times n}$, and define

$$
g(A) \equiv \max \left\{\rho(A), f\left(A-D_{A}\right)\right\}, \quad A \in \mathbb{C}^{n \times n},
$$

where $D_{A}$ is the diagonal part of $A$. Clearly $g$ is a spectrally dominant subnorm on $\mathbb{C}^{n \times n}$. Now, as in the example following Proposition 1.4, consider the matrix

$$
A_{\kappa}=I+J_{\kappa},
$$

where $J_{\kappa}$ is the $n \times n$ matrix whose upper-right entry is a real constant $\kappa$ and the rest of its entries vanish. Select $\kappa$ such that $f\left(J_{\kappa}\right)=1$. Then $g\left(A_{\kappa}\right)=1$, whereas

$$
g\left(A_{\kappa}^{m}\right)=g\left(I+m J_{\kappa}\right)=f\left(m J_{\kappa}\right)=m \underset{m \rightarrow \infty}{\longrightarrow} \infty ;
$$

so $g$ is unstable on $\mathbb{C}^{n \times n}$. Surely, $g$ is a proper subnorm on $\mathbb{C}^{n \times n}$. For if $g$ were a norm, then by its spectral dominance, the Friedland-Zenger theorem would force it to be stable on $\mathbb{C}^{n \times n}$.

\section{References}

[B] J.C. Baez, The octonions, Bull. Amer. Math. Soc. (N.S.), 39 (2002), 145-205, MR 1886087.

[BSW] F.L. Bauer, J. Stoer and C. Witzgall, Absolute and monotonic norms, Numer. Math., 3 (1961), 257-264, MR 0130104, Zbl 0111.01602.

[FZ] S. Friedland and C. Zenger, All spectral dominant norms are stable, Linear Algebra Appl., 58 (1984), 97-107, MR 0739281, Zbl 0543.15018.

[G1] M. Goldberg, A note on monotonic and semi-monotonic matrix functions, Linear and Multilinear Algebra, 24 (1989), 223-226, MR 1007257, Zbl 0703.15027.

[G2] Quasimonotonic functions on $\mathbb{C}^{n}$ and the mapping $f \rightarrow f^{+}$, Linear and Multilinear Algebra, 27 (1990), 63-71, MR 1050677, Zbl 0706.15026.

[GGL] M. Goldberg, R. Guralnick and W.A.J. Luxemburg, Stable subnorms II, Linear and Multilinear Algebra, 51 (2003), 209-219, MR 1976865. 
[GL1] M. Goldberg and W.A.J. Luxemburg, Stable subnorms, Linear Algebra Appl., 307 (2000), 89-101, MR 1741918, Zbl 0997.15021.

[GL2] _ Discontinuous subnorms, Linear and Multilinear Algebra, 49 (2001), 124, MR 1888109, Zbl 0982.15032.

[HJ] R. Horn and C.R. Johnson, Matrix Analysis, Cambridge University Press, Cambridge, 1985, MR 0832183, Zbl 0576.15001.

[W] H. Wielandt, Topics in the Analytic Theory of Matrices, Lecture Notes, Dept. of Mathematics, Univ. of Wisconsin, Madison, Wisconsin, 1967.

[Z] A.C. Zaanen, Riesz Spaces II, North Holland, Amsterdam, 1983, MR 0704021, Zbl 0519.46001.

Received September 7, 2003. Research of the first author was sponsored in part by the Fund for the Promotion of Research at the Technion, Grant 100-191.

Department of Mathematics

TEChNiON - IsRAel Institute of TEChNOLOGY

HAIFA 32000, ISRAEL

E-mail address: goldberg@math.technion.ac.il

Department of Mathematics

CAlifornia Institute of TeChNOlOgy

PASADENA, CA 91125

E-mail address: lux@caltech.edu

This paper is available via http://www.pacjmath.org/2004/215-1-3.html. 